\title{
AGT May Serve as a Prognostic Biomarker and Correlated with Immune Infiltration in Gastric Cancer
}

\author{
Fanqi Wu $\mathbb{D}^{1, *}$, Longguo Zhang $\mathbb{D}^{2, *}$, Li Wang $\mathbb{D}^{3, *}$, Dekui Zhang ${ }^{4}$ \\ 'Department of Respiratory, Lanzhou University Second Hospital, Lanzhou, Gansu Province, People's Republic of China; ${ }^{2}$ The Second Clinical Medical \\ School, Lanzhou University, Lanzhou, Gansu Province, People's Republic of China; ${ }^{3}$ Nanjing Medical University, Nanjing, Jiangsu Province, People's \\ Republic of China; ${ }^{4}$ Department of Gastroenterology, Lanzhou University Second Hospital, Lanzhou, Gansu Province, People's Republic of China
}

*These authors contributed equally to this work

Correspondence: Dekui Zhang, Department of Gastroenterology, Lanzhou University Second Hospital, No. 82, Cuiyingmen, Lanzhou, Gansu Province, People's Republic of China, Tel +86 139197886/6, Email zhangdk86I6@I26.com

Purpose: Angiotensinogen (AGT), as a component of the renin-angiotensin system (RAS), is associated with multiple risk factors for gastric cancer (GC). However, the relationship between AGT and tumor-infiltrating lymphocytes in GC remains elusive.

Methods: AGT expression was analyzed based on the Cancer Genome Atlas (TCGA) dataset. Kaplan-Meier curve was employed to assess the role of AGT expression in gastric patients' prognosis. The association between AGT expression and tumor immune infiltration was further evaluated via exploring Tumour Immune Estimation Resource (TIMER) and The Gene Expression Profiling Interactive Analysis (GEPIA). We also used multiple public databases to analyse the aberrant methylation of AGT, construct proteinprotein interaction (PPI) and gene ontology (GO) analyses.

Results: AGT was overexpressed in GC tissues compared with normal gastric tissues $(P<0.05)$. High AGT expression related with poorer overall survival of patients with GC, especially in advanced GC patients. Immune infiltration analysis revealed that AGT was associated with several immune cells (including B cells, CD4+ T cells, macrophages), and AGT expression was also associated with the markers of NK cells, TAMs, Tregs, and so on (all $P<0.05$ ). Methylation analysis indicated that hypomethylation may lead to abnormal upregulation of the AGT. GO analysis showed that AGT and its related genes were enriched in systemic arterial blood pressure by hormone, regulation of blood volume by renin-angiotensin, NIK/NF-kappaB signaling, ficolin-1-rich granule and so on. Conclusion: AGT could act as a promising biomarker for prognosis and immune infiltration in GC.

Keywords: angiotensinogen, prognosis, tumor-infiltrating, methylation, stomach adenocarcinoma

\section{Introduction}

GC ranks the fifth most frequently occurring cancer and the fourth leading cause of cancer-related death, ${ }^{1}$ in 2020. Common risk factors for GC include high salt intake, infection with Helicobacter pylori bacteria, smoking and age. ${ }^{2}$ Currently, the diagnosis of gastric cancer relies on the use of upper gastrointestinal endoscopic biopsy and standard serum biomarkers, comprising $\alpha$-fetoprotein (AFP), carcinoembryonic antigen (CEA), pepsinogen, and carbohydrate antigens (CA): CA19-9. ${ }^{3-5}$ However, these diagnostic methods have many shortcomings, for example, endoscopy is a discomfort to the patient and costly invasive technique, and standard serum biomarkers have poor sensitivity and specificity. ${ }^{6-8}$ Therefore, GC is characterized by late diagnosis and poor prognosis. It is necessary for us to find reliable biomarkers for diagnosing early GC and predicting the prognosis of advanced GC.

Tumor microenvironment (TME), consisting of a vast variety of innate and adaptive immune cells and non-immune stromal cells such as endothelial cells and mesenchymal cells, profoundly influences cancer progression and therapeutic responses. ${ }^{9-11}$ Currently, immunotherapy has made revolutionary breakthroughs in the treatment of cancer. However, immunotherapy faces many problems, for example, limited number of patients achieve survival benefits from it in clinic, 
and treatment resistance. ${ }^{12,13}$ Further investigations of the relationship between TME, particularly tumor immune microenvironment, and GC may help to address these limitations. Thus, this requires us to look for more potential immune infiltration-related markers.

AGT, produced mainly by the liver, is an essential component of the RAS. ${ }^{14}$ AGT is cleaved by renin enzyme to produce angiotensin 1 (Ang I) when blood pressure is reduced, and AGT-converting enzyme (ACE) splits Ang I to generate angiotensin 2 (Ang II). ${ }^{15,16}$ RAS plays an important role in maintaining fluid and electrolyte homeostasis, ${ }^{17}$ and RAS irregulated expression leads to kidney disease, heart failure, hypertension, and diabetes. ${ }^{18,19}$ Interestingly, RAS is also involved in the regulation of TME, such as resistance to apoptosis, promoting tumor angiogenesis, and involvement in inflammation, migration, invasion and metastasis. ${ }^{17,20}$ However, previous studies have concentrated on the association between AGT gene polymorphism and GC risk factors, ${ }^{21}$ such as high-salt diet, and Helicobacter pylori infection. As an important member of the RAS, the role of AGT in the TME of GC is still unknown.

In this study, hub genes in GC were filtered based on the TCGA dataset, and AGT was selected for further analysis. We used several public databases to evaluate the prognostic and diagnostic values of AGT expression in GC. We also investigated the correlation between AGT expression and immune infiltration levels of GC. In addition, these results will help us to understand the connection between AGT expression and TME in GC.

\section{Materials and Methods}

\section{Gene Expression Data Extraction}

The primitive data of stomach adenocarcinoma (STAD) were acquired from TCGA (https://portal.gdc.cancer.gov/) database. We further investigated differentially expressed mRNAs through "Limma" package (version: 3.40.2) of $\mathrm{R}$ software. Adjusted P values were used to rectify false-positive outcomes in TCGA, and we also considered the thresholds for filtering hubs genes as follows: Adjusted $P<0.05$ and $\mid \log 2$ (fold change) $\mid \geq 2$. We divided the GC patients into two subgroups, and the cut-off value was the median AGT expression level.

\section{Immune Infiltration Analysis}

TIMER (https://cistrome.shinyapps.io/timer/) database was utilized to explore the potential crosstalk among AGT expression levels and the abundance of infiltrating immune cells (such as CD8+ T cells, dendritic cells, CD4+ T cells, macrophages, B cells, and neutrophils) through tumor purity-corrected partial Spearman correlation method. We further examined the prognostic value of these immune cells in GC using Kaplan-Meier curve. In addition, we estimated the correlation of immune cell infiltration level with AGT copy numbers. Moreover, we studied the connection among AGT expression level and the biomarkers of tumor-infiltrating immune cells (including biomarkers of TAM, B cell, T-helper 17 (Th17) cell, T-helper 1 (Th1) cell, T-helper 2 (Th2) cell, CD8+ T cell, CD4+ T cell, Treg, exhausted T cell, follicular helper T (Tfh) cell, M1 macrophage, M2 macrophage, dendritic cell, neutrophil, natural killer (NK) cell, and monocyte) by Spearman correlation.

\section{GEPIA Database Analysis}

GEPIA database (http://gepia.cancer-pku.cn/), based on TCGA dataset, was applied to analyze the expression distribution of AGT among GC tissues and normal gastric tissues. Then, GEPIA was also applied to assess the prognostic value of AGT in GC, and indicators to assess prognosis including overall survival (OS) and recurrence-free survival/disease-free survival (RFS/DFS). Furthermore, GEPIA was employed to verify the correlated gene biomarkers (including biomarkers of dendritic cells, B cells, Tregs, Th2 cells, CD4+ T cells, and TAMs) in the TIMER analysis through Spearman correlation method (X-axis: AGT expression levels, Y-axis: biomarkers of tumor-infiltrating immune cells expression levels, $P<0.05$ was considered statistically significant).

\section{Methylation Analysis of AGT}

In order to estimate the possible values of AGT in GC from an epigenetic perspective, linear regression analysis was employed to estimate the correlation of the AGT mRNA expression with the AGT DNA methylation level. Furthermore, DiseaseMeth version 2.0 (http://bio-bigdata.hrbmu.edu.cn/diseasemeth/) database was used to analyze methylation levels 
of AGT among the GC tissues and normal tissues. Then, we used MethSurv (https://biit.cs.ut.ee/methsurv/) to explore the connection among hub gene's expression and their DNA methylation status of $\mathrm{CpG}$ island and to evaluate the potential role of between each $\mathrm{CpG}$ site and prognosis of GC.

\section{PPI and GO Analysis}

In order to elucidate potential interactions with AGT, The Search Tool for the Retrieval of Interacting Genes (STRING, https://string-db.org/) was applied to build PPI network. In addition, GeneMANIA database (http://genemania.org/) was also used to predict the relationship between AGT and its functionally similar genes. AGT and AGT-related genes, which were identified by STRING and GeneMANIA PPI networks, were included in the GO function analyses by using Metascape database (https://metascape.org/gp/index.html).

\section{Statistical Analyses}

OS and RFS/DFS were used to evaluate the GC patients' prognosis between the low/high AGT expression groups based on the Kaplan-Meier method, via the "survival" R package. The hazard ratio (HR), 95\% confidence intervals (CI) and logrank P value were evaluated. Kaplan-Meier plotter (http://kmplot.com/analysis/) was employed to study the link between AGT expression and GC patients' prognosis. Receiver operating characteristic (ROC) curve was employed to estimate the ability of AGT to diagnose GC by the "ROCR" R package.

\section{Results}

\section{AGT Was Overexpression in GC Tissues}

A total of 405 samples (including $373 \mathrm{GC}$ samples and 32 normal control samples) were included in our study. There were 1877 differentially expressed genes (DEGs) filtered from the TCGA data set, including 898 upregulated and 979 downregulated genes. Among these DEGs, we chose AGT for further analysis. And we found that the expression of AGT was revealed to be higher in GC samples than in normal samples in TCGA dataset ( $\operatorname{logFC}=2.13$ ) (Figure 1A). To certify the result in TCGA, GEPIA was applied to investigate the expression of AGT among GC tissues and normal tissues; we found that the AGT was upregulated in GC tissues (Figure 1B). Moreover, we constructed an ROC to confirm the diagnostic value of AGT upregulation for GC, and the area under the receiver operating characteristic curve (AUC) was $0.717(P<0.0001)$ (Figure 1C).

\section{AGT Overexpression Correlated with Poor Prognosis in GC}

The potential prognosis value between AGT and GC was firstly investigated through Kaplan-Meier plotter method based on the TCGA database. The results showed that AGT upregulation was significantly correlated with poor OS (logrank $P=0.006$ ) (Figure 1D), while it has no significant correlations with RFS/DFS (logrank $P=0.231$ ) (Figure 1E). Furthermore, the prognostic value of AGT expression was verified using GEPIA and Kaplan-Meier plotter, results showed that upregulated AGT expression was significantly correlated with poor OS (GEPIA, logrank $P=0.020$, Figure 1F; Kaplan-Meier plotter, logrank $P=0.003$, Figure $1 \mathrm{G}$ ). Similarly, it has no significant association with RFS/ DFS (GEPIA, logrank $P=0.07$, Figure 1H; Kaplan-Meier plotter, logrank $P=0.11$, Figure 1I).

Based on the TNM stage, we divided all patients into the TCGA database into two sets: TNM stage I/II, TNM stage III/IV. There was no correlation between AGT expression and OS in TNM stage I/II group (logrank $P=0.217$ ) (Figure $\underline{\mathrm{S} 1 \mathrm{~A}}$ ). Interestingly, AGT overexpression was an independent risk factor for OS in TNM stage III/IV group (logrank $P=0.012$ ) (Figure S1B). In addition, GEPIA was utilized to validate the results. Consistent with these results, we found that there was no correlation between AGT expression and OS in early stages (stage I: logrank $P=0.058$, Figure S1C; stage II: logrank $P=0.210$, Figure S1D). Nevertheless, upregulated AGT was significantly related to poorer prognosis of OS in TNM stage III/IV group (stage III: logrank $P=0.041$, Figure S1E; stage IV: logrank $P=0.013$, Figure S1F). 

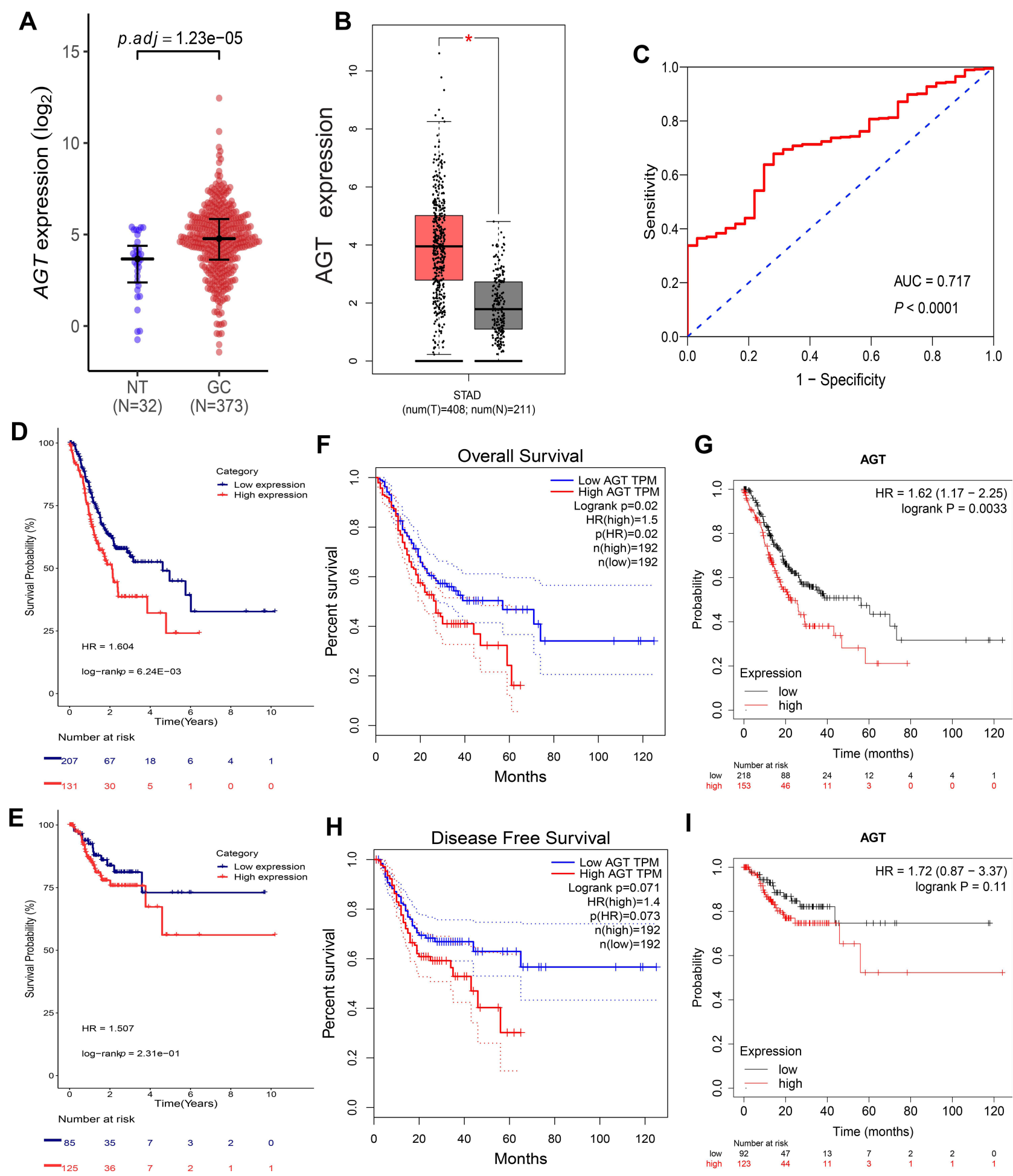

Figure I AGT expression in GC tissues and normal tissues based on TCGA database (A) and GEPIA database (B). The diagnostic value of AGT in GC using ROC curve (C). Correlation between AGT expression level and OS (D) and RFS/DFS (E) in GC based on TCGA dataset. Correlation between AGT expression and OS in GC based on GEPIA database (F), Kaplan-Meier Plotter (G); Correlation between AGT expression and RFS/DFS based on GEPIA database (H), Kaplan-Meier Plotter (I).

Notes: $P$-value Significant Codes: $*$ Stands for $P<0.05$.

Abbreviations: P.adj, adjusted P; NT, normal tissue; GC, gastric cancer; STAD, stomach adenocarcinoma; AUC, the area under the receiver operating characteristic curve; HR, hazard ratio. 


\section{Correlation Between AGT Expression and Immune Infiltration}

To investigate the relationship between AGT expression levels and immune infiltration levels in GC, TIMER database was used to perform the following analysis. Our results showed that there was a significant association between AGT gene copy number alterations and immune cell infiltration levels, such as macrophages, CD8+ $\mathrm{T}$ cells, neutrophil, dendritic cells, B cells, and CD4+ T cells in GC (Figure 2A). Moreover, Kaplan-Meier plots were used to explore the impact of AGT expression and immune infiltration on the OS of GC patients. We found that AGT expression $(P=0.003)$ and macrophage infiltration $(P=0.004)$ were significantly correlated with prognosis (Figure 2B). We further investigated whether the expression of AGT was connected with the infiltration of immune cells. Our results demonstrated that AGT expression was positively correlated with infiltration of $\mathrm{B}$ cells $(\mathrm{r}=0.124, P=1.70 \mathrm{e}-02), \mathrm{CD} 4+\mathrm{T}$ cells $(\mathrm{r}=0.314, P=8.08 \mathrm{e}-10)$, and macrophages $(\mathrm{r}=0.27, P=1.30 \mathrm{e}-$ 07) (Figure 2C). Our results suggested that AGT expression was significantly correlated with immune infiltration in GC.

\section{Relationship Between AGT and Immune Cell Biomarkers}

We further illustrated the relation between AGT and biomarkers of immunocytes in GC using TIMER and GEPIA. The correlation of AGT with markers of immunocytes was first analyzed by TIMER, and adjusted for tumor purity. The results demonstrated that AGT was significantly correlated with diversity immune cells biomarkers, including biomarkers of $\mathrm{B}$ cell (CD19, CD79A), CD8 $+\mathrm{T}$ cell (CD8B), CD4 $+\mathrm{T}$ cell (CD4), neutrophil cell (ITGAM, CCR7), dendritic cell (CD1C, CD1C), monocyte cell (CSF1R), TAM (CCL2, CD68, IL10), M1 macrophage (NOS2), M2 macrophage (MS4A4A), Th1 cell (STAT4), Th2 cell (STAT6, STAT5A), Tfh cell (BCL6), Th17 cell (STAT3), Treg (FOXP3, CCR8, STAT5B, TGFB1), and NK cell (KIR2DL4, KIR2DS4) (Table 1, Figure 3A-I). Meanwhile, GEPIA was surveyed to analyzed the correlation between AGT expression and the markers of B cell, CD4+ T cell, dendritic cell, TAM, M2 macrophage, Th2 cell, Treg, NK cell. Consistently with the results of TIMER data analysis, we found that there was significantly positive of AGT with those immune cell biomarkers in GC, while it was negatively correlated with NK cell's biomarkers (Table 2).

\section{Correlation Between Methylation and Expression of AGT}

To elucidate the potential mechanisms of aberrant AGT expression in GC, we firstly analyzed the relationship between AGT expression and its DNA methylation status based on TCGA database. And our results showed that AGT upregulation was significantly negatively connected with its total DNA methylation levels (cor $=-0.41$, $P=2.2 \mathrm{e}-16$ ) (Figure 4A). Additionally, we used DiseaseMeth version 2.0 database to explore methylation levels among GC samples and paracancerous samples. The results illustrated that the methylation levels of AGT were higher in adjacent normal samples compared with GC samples; however, there was no statistical significance between the two groups $(P=3.786 \mathrm{e}-01)$ (Figure 4B). And our results illustrated that expression level of AGT was associated with six CpG sites (such as cg07502417, cg24474852, cg22647018, cg26882410, cg14523948, and cg27401395) in the AGT DNA sequences (Figure 4C). Kaplan-Meier plots showed that four CpG sites (cg07502417, cg22647018, cg14523948, and cg27401395) were correlated with favorable prognosis (Figure 4D-I).

\section{PPI and AGT-Related Signaling Pathways}

Based on the STRING database, we constructed a PPI network for AGT to explore the potential interactions among it. There were 11 nodes and 43 edges in this PPI network. We also found that AGT was co-expressed with ACE, CMA1, REN, AGTR1, AGTR2, ATP6AP2, ACE2, ENPEP, EDN1, and PRG2 (Figure 5A). Furthermore, GeneMANIA database was employed to create a PPI network for AGT and to explore its coregulated hub genes. The results illustrated that AGT was co-expressed with REN, AGTR2, AGTR1, ENPEP, ACE2, CMA1, JUND, MAS1, SERPIND1, ACE, PRG2, ANPEP, PTP4A3, SERPINA3, SERPINA7, ATP6AP2, CPA3, SERPINA1, 


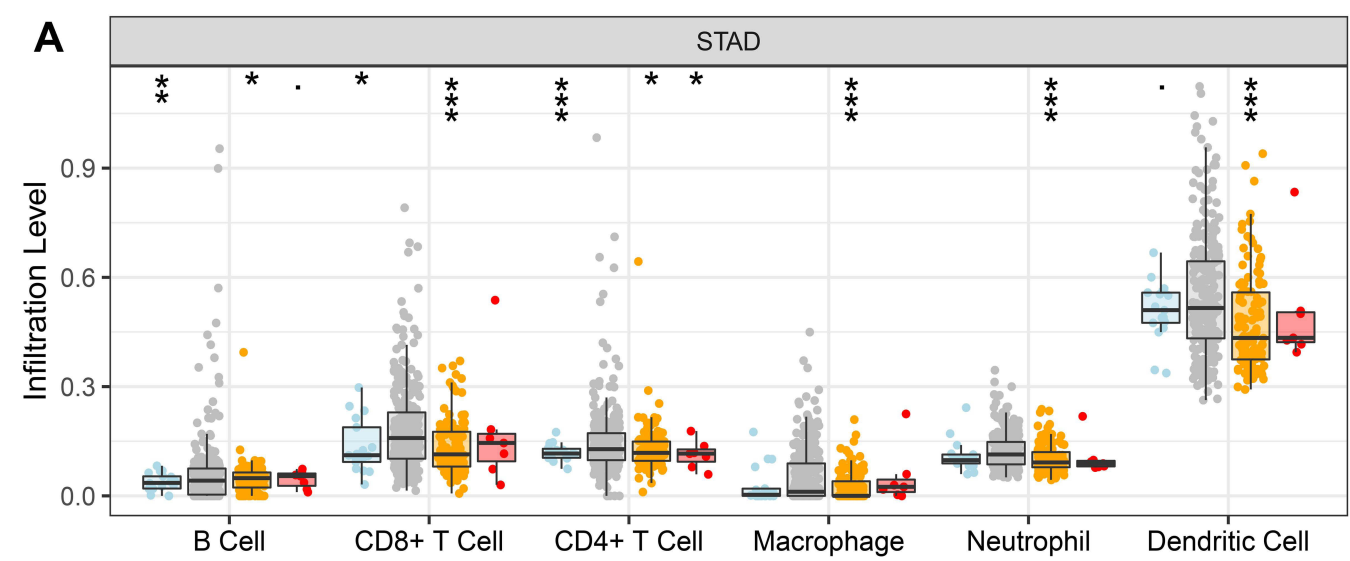

Copy Number

亩 Arm-level Deletion

Diploid/Normal

由 Arm-level Gain

稙 High Amplication

B
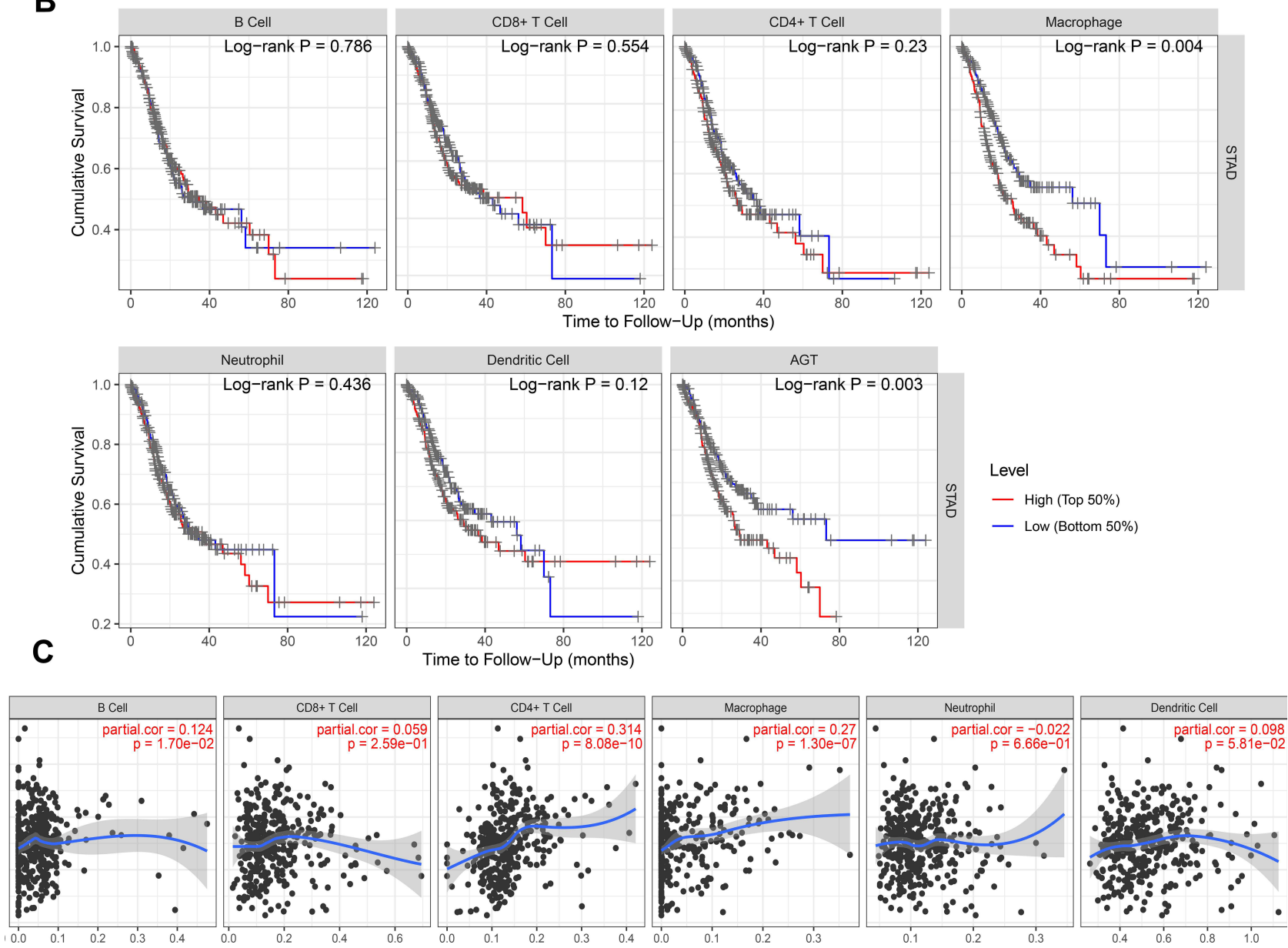

Figure 2 (A) The relationship between AGT gene copy number alterations and immune cell infiltration levels. (B) Prognostic value of immune cells and AGT in GC. (C) Correlation of AGT expression with immune infiltration level in GC.

Notes: $P$-value Significant Codes: $*$ Stands for $P<0.05$, **Stands for $P<0.01$, ***Stands for $P<0.00$ I.

Abbreviation: STAD, stomach adenocarcinoma.

APOH, TUB (Figure 5B). GO enrichment analysis was performed to evaluate the biological functions of AGT and its related genes, and it was found that these genes were mainly enriched in regulation of systemic arterial blood pressure by hormone and regulation of blood volume by renin-angiotensin (Figure 5C). 
Table I Correlation Analysis Between AGT and Related Genes and Markers of Immune Cells in TIMER

\begin{tabular}{|c|c|c|c|c|c|c|c|}
\hline \multirow[t]{2}{*}{ Description } & \multirow[t]{2}{*}{ Gene Markers } & \multicolumn{3}{|c|}{ None } & \multicolumn{3}{|c|}{ Purity } \\
\hline & & $\mathbf{R}$ & $P$ value & P Star & $\mathbf{R}$ & $P$ value & P Star \\
\hline \multirow[t]{2}{*}{ B cell } & CDI9 & 0.24 & $7.5 \mathrm{E}-07$ & $* * *$ & 0.237 & $2.9 \mathrm{E}-06$ & $* * *$ \\
\hline & CD79A & 0.208 & 2.IE-05 & $* * *$ & 0.205 & $6.0 \mathrm{E}-05$ & $* * *$ \\
\hline \multirow[t]{2}{*}{$\mathrm{CD} 8+\mathrm{T}$ cell } & CD8A & 0.056 & $2.6 \mathrm{E}-0 \mathrm{I}$ & - & 0.063 & $2.2 \mathrm{E}-0 \mathrm{I}$ & - \\
\hline & CD8B & 0.113 & 2.IE-02 & $*$ & 0.124 & $1.6 \mathrm{E}-02$ & $*$ \\
\hline CD4+ $\mathrm{T}$ cell & $\mathrm{CD} 4$ & 0.182 & $1.9 \mathrm{E}-04$ & $* * *$ & 0.183 & $3.3 \mathrm{E}-04$ & $* * *$ \\
\hline \multirow[t]{3}{*}{ MI macrophage } & NOS2 & 0.116 & $1.8 \mathrm{E}-02$ & $*$ & 0.102 & 4.7E-02 & $*$ \\
\hline & IRF5 & 0.090 & $6.6 \mathrm{E}-02$ & - & 0.091 & $7.7 \mathrm{E}-02$ & - \\
\hline & PTGS2 & 0.086 & $7.9 \mathrm{E}-02$ & - & 0.078 & I.3E-0| & - \\
\hline \multirow[t]{3}{*}{ M2 macrophage } & CDI63 & 0.010 & $4.2 \mathrm{E}-02$ & $*$ & 0.087 & $9.0 \mathrm{E}-02$ & - \\
\hline & VSIG4 & 0.094 & $5.5 \mathrm{E}-02$ & - & 0.087 & $9.1 E-02$ & - \\
\hline & MS4A4A & 0.132 & $7.0 \mathrm{E}-03$ & $* *$ & 0.125 & $1.5 \mathrm{E}-02$ & $*$ \\
\hline \multirow[t]{3}{*}{ Neutrophil } & CEACAM8 & 0.080 & I.0E-0I & - & 0.085 & $9.8 \mathrm{E}-02$ & - \\
\hline & ITGAM & 0.123 & I.2E-02 & $*$ & 0.115 & $2.6 \mathrm{E}-02$ & $*$ \\
\hline & CCR7 & 0.283 & 5.IE-09 & $* * *$ & 0.294 & $5.6 \mathrm{E}-09$ & $* * *$ \\
\hline \multirow[t]{7}{*}{ Dendritic cell } & HLA-DPBI & 0.016 & $7.5 \mathrm{E}-0 \mathrm{I}$ & - & 0.009 & $8.6 \mathrm{E}-0 \mathrm{I}$ & - \\
\hline & HLA-DQBI & -0.053 & $2.8 \mathrm{E}-0 \mathrm{I}$ & - & -0.072 & $1.6 \mathrm{E}-0 \mathrm{I}$ & - \\
\hline & HLA-DRA & -0.067 & $1.8 \mathrm{E}-0 \mathrm{I}$ & - & -0.070 & I.7E-0I & - \\
\hline & HLA-DPAI & -0.015 & $7.5 \mathrm{E}-0 \mathrm{I}$ & - & -0.022 & $6.8 \mathrm{E}-0 \mathrm{I}$ & - \\
\hline & CDIC & 0.365 & $1.5 \mathrm{E}-14$ & $* * *$ & 0.371 & $7.6 \mathrm{E}-14$ & $* * *$ \\
\hline & NRPI & 0.268 & $3.2 \mathrm{E}-08$ & $* * *$ & 0.249 & $9.4 \mathrm{E}-07$ & $* * *$ \\
\hline & ITGAX & 0.110 & $2.5 \mathrm{E}-02$ & $*$ & 0.085 & $9.8 \mathrm{E}-02$ & - \\
\hline \multirow[t]{2}{*}{ Monocyte } & CD86 & 0.083 & $9.2 \mathrm{E}-02$ & - & 0.066 & $2.0 \mathrm{E}-0 \mathrm{I}$ & - \\
\hline & CDII5(CSFIR) & 0.261 & $7.5 \mathrm{E}-08$ & $* * *$ & 0.243 & $1.7 \mathrm{E}-06$ & $* * *$ \\
\hline \multirow[t]{3}{*}{ TAM } & $\mathrm{CCL} 2$ & 0.203 & $3.2 \mathrm{E}-05$ & $* * *$ & 0.177 & $5.4 \mathrm{E}-04$ & $* * *$ \\
\hline & CD68 & 0.159 & I.2E-03 & $* *$ & 0.136 & $8.0 \mathrm{E}-03$ & $* *$ \\
\hline & ILIO & 0.145 & $3 . I E-03$ & $* *$ & 0.120 & $1.9 \mathrm{E}-02$ & $*$ \\
\hline \multirow[t]{4}{*}{ Thl cell } & T-bet (TBX2I) & 0.025 & 6.IE-0I & - & 0.023 & $6.6 \mathrm{E}-0 \mathrm{I}$ & - \\
\hline & STAT4 & 0.201 & 3.7E-05 & $* * *$ & 0.201 & $7.9 \mathrm{E}-05$ & $* * *$ \\
\hline & STATI & -0.070 & $1.5 \mathrm{E}-0 \mathrm{I}$ & - & -0.075 & I. $4 \mathrm{E}-0 \mid$ & - \\
\hline & TNF- $\alpha$ (TNF) & -0.030 & $5.4 \mathrm{E}-0 \mathrm{I}$ & - & -0.043 & $4.0 \mathrm{E}-0 \mathrm{I}$ & - \\
\hline \multirow[t]{3}{*}{ Th2 cell } & STAT6 & 0.193 & $7.3 \mathrm{E}-05$ & $* * *$ & 0.190 & $2.0 \mathrm{E}-04$ & $* * *$ \\
\hline & STAT5A & 0.136 & $5.6 \mathrm{E}-03$ & $* *$ & 0.144 & $5.1 \mathrm{E}-03$ & $* *$ \\
\hline & $\mathrm{ILI} 3$ & 0.018 & $7.2 \mathrm{E}-0 \mathrm{I}$ & - & 0.009 & $8.6 \mathrm{E}-0 \mathrm{I}$ & - \\
\hline \multirow[t]{2}{*}{ Tfh cell } & BCL6 & 0.117 & $1.7 \mathrm{E}-02$ & $*$ & 0.109 & $3.4 \mathrm{E}-02$ & $*$ \\
\hline & IL2I & -0.035 & $4.7 \mathrm{E}-0 \mathrm{I}$ & - & -0.042 & 4.IE-0I & - \\
\hline \multirow[t]{2}{*}{ ThI7 cell } & STAT3 & 0.179 & $2.5 \mathrm{E}-04$ & $* * *$ & 0.174 & $6.4 \mathrm{E}-04$ & $* * *$ \\
\hline & ILI7A & 0.069 & $1.6 \mathrm{E}-0 \mathrm{I}$ & - & 0.068 & $1.9 \mathrm{E}-01$ & - \\
\hline \multirow[t]{4}{*}{ Treg } & FOXP3 & 0.175 & $3.5 \mathrm{E}-04$ & $* * *$ & 0.176 & $5.7 \mathrm{E}-04$ & $* * *$ \\
\hline & CCR8 & 0.179 & $2.5 \mathrm{E}-04$ & $* * *$ & 0.170 & $8.9 \mathrm{E}-04$ & $* * *$ \\
\hline & STAT5B & 0.354 & $1.4 \mathrm{E}-13$ & $* * *$ & 0.342 & $7.2 E-12$ & $* * *$ \\
\hline & TGF $\beta$ (TGFBI) & 0.232 & $1.8 \mathrm{E}-06$ & $* * *$ & 0.206 & $5.2 \mathrm{E}-05$ & $* * *$ \\
\hline \multirow[t]{4}{*}{ T cell exhaustion } & PD-I (PDCDI) & -0.006 & $9.0 \mathrm{E}-01$ & - & 0.001 & $9.9 \mathrm{E}-01$ & - \\
\hline & CTLA4 & $-0.04 \mid$ & 4.IE-0I & - & -0.043 & 4.IE-0I & - \\
\hline & LAG3 & -0.059 & $2.3 \mathrm{E}-0 \mathrm{I}$ & - & -0.054 & $2.9 \mathrm{E}-0 \mathrm{I}$ & - \\
\hline & TIM-3 (HAVCR2) & 0.036 & $4.6 \mathrm{E}-0 \mathrm{I}$ & - & 0.023 & $6.5 \mathrm{E}-0 \mathrm{I}$ & - \\
\hline
\end{tabular}

(Continued) 
Table I (Continued).

\begin{tabular}{|c|c|c|c|c|c|c|c|}
\hline \multirow[t]{2}{*}{ Description } & \multirow[t]{2}{*}{ Gene Markers } & \multicolumn{3}{|c|}{ None } & \multicolumn{3}{|c|}{ Purity } \\
\hline & & $\mathbf{R}$ & $P$ value & P Star & $\mathbf{R}$ & $P$ value & P Star \\
\hline \multirow[t]{7}{*}{ NK cell } & KIR2DLI & -0.053 & $2.9 \mathrm{E}-0 \mathrm{I}$ & - & -0.06 & $2.5 \mathrm{E}-0 \mathrm{I}$ & - \\
\hline & KIR2DL3 & -0.007 & $1.5 \mathrm{E}-0 \mathrm{I}$ & - & -0.009 & 6.7E-0I & - \\
\hline & KIR2DL4 & -0.207 & 2.IE-05 & $* * *$ & -0.210 & $3.9 \mathrm{E}-05$ & $* * *$ \\
\hline & KIR3DLI & -0.070 & $1.5 \mathrm{E}-0 \mathrm{I}$ & - & -0.081 & I.IE-0I & - \\
\hline & KIR3DL2 & -0.063 & $2.0 \mathrm{E}-0 \mathrm{I}$ & - & -0.078 & $1.3 \mathrm{E}-0 \mathrm{I}$ & - \\
\hline & KIR3DL3 & -0.091 & $6.4 \mathrm{E}-02$ & - & -0.018 & $2.2 \mathrm{E}-02$ & - \\
\hline & KIR2DS4 & -0.105 & $3.2 \mathrm{E}-02$ & $*$ & -0.108 & $3.6 \mathrm{E}-02$ & $*$ \\
\hline
\end{tabular}

Notes: P-value Significant Codes: $-P \geq 0.05, * P<0.05$, ** $P<0.01$, *** $P<0.001$.

\section{Discussion}

$\mathrm{GC}$ is one of the most common and deadly neoplasms affecting individuals globally. ${ }^{1}$ Improvements in surgery, radiotherapy, chemotherapy, targeted therapy and immunotherapy have significantly decreased the mortality of GC patients. ${ }^{22}$ However, there are still patients undergoing relapse and progress with similar treatment. Thus, there was a clear need to identify novel potential predictive and prognostic markers in patients with GC. Previous studies have shown that AGT is upregulated in GC and indicated poor prognosis in GC. ${ }^{16,23,24}$ Liu et al showed that AGT expression in exosomes positively correlates with serum level in GC. ${ }^{23} \mathrm{Li}$ et al reported that AGT overexpression is associated with poor prognosis in diffuse-type GC. ${ }^{16}$ Our conclusions were consistent with the previous studies that upregulation of AGT is associated with poorer OS in GC patients. Zhang et al observed that there is no association between AGT expression and TNM stage. ${ }^{24}$ Interestingly, we found that TNM stage influenced the correlation between AGT expression and OS of GC patients. For example, AGT could serve as a prognostic biomarker in TNM stage III/IV GC patients. However, no correlation was observed between AGT expression and prognosis in TNM stage I/II GC patients. Moreover, we explored the association between AGT expression and RFS/DFS in GC. Unfortunately, no association was found between them. Collectively, our results emphasized that AGT might be a valid biomarker for OS in advanced GC.

Increasing evidence has shown that tumor-infiltrating immune cells induce an immune microenvironment and significantly influence both the growth and evolution of neoplasms and therapeutic response. ${ }^{25-27}$ Immune microenvironment also acts as a key regulator of progression in GC. Thus, we evaluated the correlation between the expression of AGT and tumor-infiltrating immune cells. Results revealed that AGT expression was positively associated with diverse tumor-infiltrating immune cells in GC, including macrophages, CD4+ T cells, and B cells. To better understand why AGT connects with high immune cell infiltration and predicts poor OS in gastric cancer, we investigated the relationship between AGT expression levels and immune cell biomarkers. We found that AGT was negatively correlated with NK cell's biomarkers (KIR2DL4, KIR2DS4). NK cells dysfunction associates with advanced disease progression in solid tumor. ${ }^{28} \mathrm{Li}$ et al showed that high tumor-infiltrating NK cells density indicates poor prognosis in GC. ${ }^{29}$ Besides, AGT was positively correlated with biomarkers of TAMs (especially M2 macrophages), Tregs. TAMs promote tumor initiation, progression, metastasis, ${ }^{30}$ and immune suppression. ${ }^{31}$ M2 macrophages act as immunosuppressive innate immune cells, ${ }^{32}$ and relate to worse prognosis in malignant tumors. ${ }^{33}$ Moreover, M2 macrophages are found to be able to promote tumor growth, progression, invasion and epithelial mesenchymal transition (EMT) through secreting anti-inflammatory cytokines. ${ }^{34,35}$ Tregs are able to inhibit immune responses and promote immune escape, tumor growth and invasion. ${ }^{36}$ To summarize, our results suggest that the crosstalk between AGT overexpression and immune cells (including NK cells, TAMs, Tregs) could promote cancer progression and lead to poorer prognosis in GC.

Epigenetic modifications, particularly DNA methylation, occupy an important role in the induction and progression of human disease, ${ }^{37}$ including GC. ${ }^{38}$ DNA methylation could lead to dysregulation of tumor 
A Dendritic cell

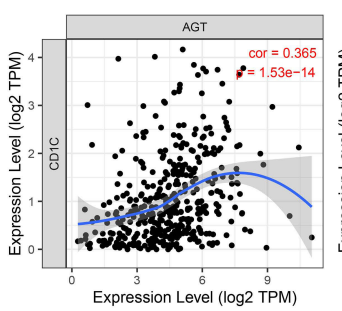

E Th2 cell

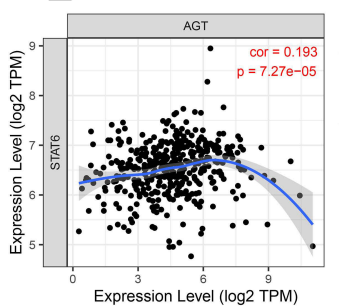

H TAM

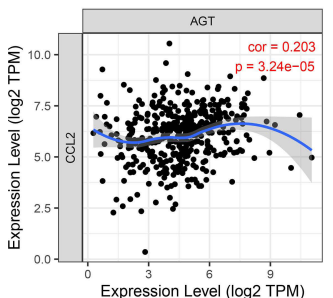

$\mathbf{K}$ Treg

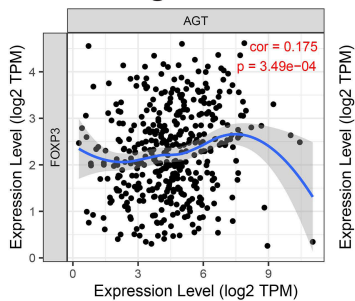

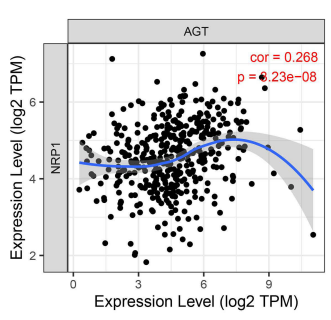

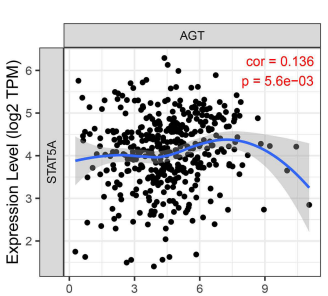

Expression Level $(\log 2$ TPM)

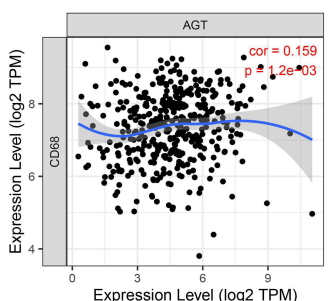

\begin{tabular}{ccc}
$\frac{1}{3}$ & $\dot{1}$ & \multicolumn{1}{c}{} \\
Expression Level & $(\log 2$ & TPM)
\end{tabular}

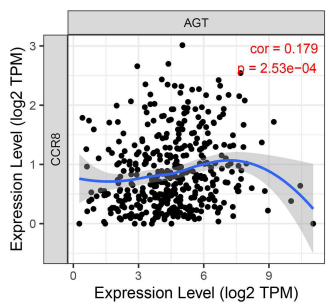

B Th17 cell

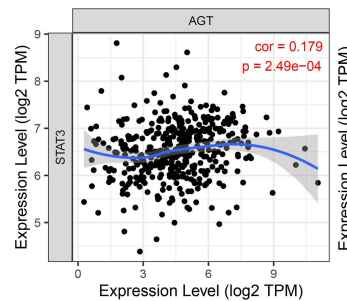

F B cell

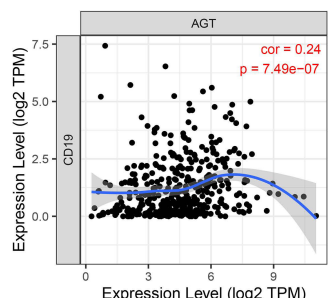

Expression Level $\stackrel{6}{{ }^{3}} \stackrel{9}{9} 2$ TPM)

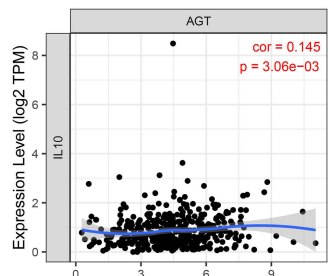

$\begin{array}{cc}3 & 6 \\ \text { Expression Level } & 9 \\ (\log 2 & 9 \\ \text { TPM) }\end{array}$

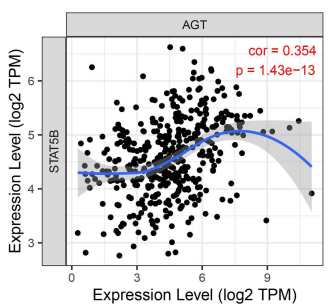

C NK cell

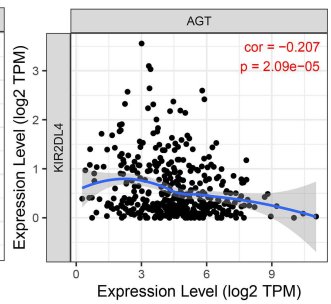

$\stackrel{3}{3} \stackrel{6}{6} \stackrel{9}{9}$

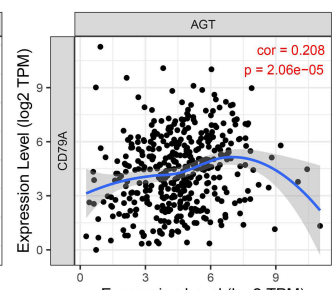

I Expression Level (log2
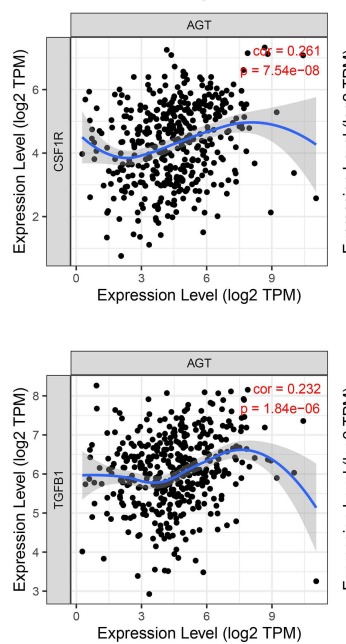

D CD4+ T cell

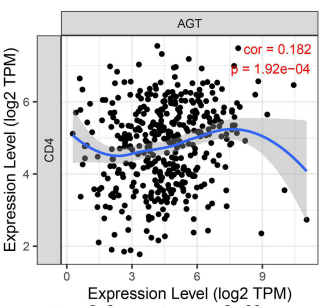

G Neutrophil
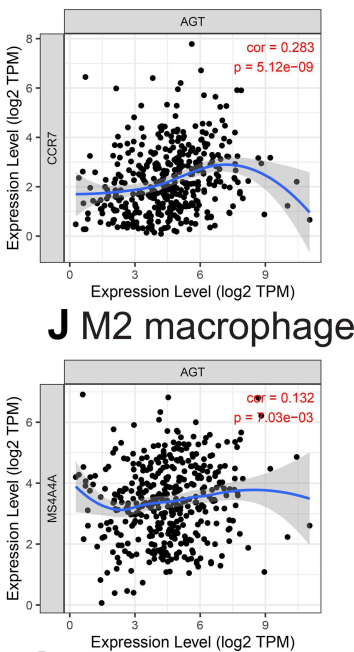

L Th1 cell

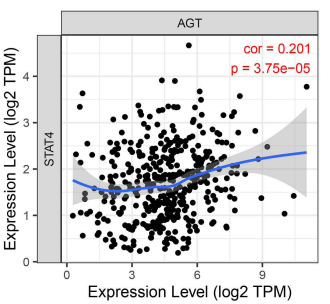

Figure 3 Immune cell markers associated with AGT (P<0.0I), including (A) Dendritic cell; (B) ThI7 cell; (C) NK cell; (D) CD4+ T cell; (E) Th2 cell; (F) B cell; (G) Neutrophil; (H) TAM; (I) Monocyte; (J) M2 macrophage; (K) Treg; (L) ThI cell.

suppressor genes and oncogenes. ${ }^{39}$ Zhang et al demonstrated that AGT is aberrantly methylated and overexpressed in GC. ${ }^{24}$ In our research, we further studied the regulatory mechanism of AGT expression in gastric cancer. Consistent with previous research, we found that AGT was inversely related to DNA methylation levels in GC. Nevertheless, there was no difference in the methylation level of AGT between GC and normal samples; this might be caused by the small size of the normal samples. DNA methylation analysis illustrated that all CpGs with both higher and lower methylation levels were found in Open_Sea. Consequently, high levels of methylation of CpG sites (cg07502417, cg22647018, cg14523948, and $\operatorname{cg} 27401395)$ were positively significantly associated with better prognosis. In summary, AGT was negatively modulated by AGT methylation, and its methylation status might act as a promising biomarker for prognosis prediction of GC.

In the GO analysis, we found that AGT and AGT-related genes were enriched in multiple pathways, including not only the RAS-related pathways (systemic arterial blood pressure by hormone, regulation of blood volume by reninangiotensin) but also NIK/NF-kappaB signaling, ficolin-1-rich granule, etc. NIK/NF-kappaB signaling pathway may lead to oncogenesis, and progression through regulating EMT in $\mathrm{GC}^{40}$ Our results also demonstrated that upregulated of AGT was positively connected with biomarkers of M2 macrophages. M2 macrophages infiltration in GC is related to metastasis via 
Table 2 Correlation Analysis Between AGT and Related Genes and Markers of Immune Cells in GEPIA

\begin{tabular}{|c|c|c|c|c|c|c|c|}
\hline \multirow[t]{2}{*}{ Description } & \multirow[t]{2}{*}{ Gene Markers } & \multicolumn{3}{|c|}{ Tumour } & \multicolumn{3}{|c|}{ Normal } \\
\hline & & $\mathbf{R}$ & $P$ value & P Star & $\mathbf{R}$ & $P$ value & P Star \\
\hline \multirow[t]{2}{*}{$B$ cell } & CDI9 & 0.21 & $2.9 \mathrm{E}-05$ & $* * *$ & 0.68 & 5.IE-06 & $* * *$ \\
\hline & CD79A & 0.17 & 4.7E-04 & $* * *$ & 0.61 & I.0E-04 & $* * *$ \\
\hline CD4+ T cell & CD4 & 0.16 & $8.2 \mathrm{E}-04$ & $* * *$ & 0.54 & $7.2 \mathrm{E}-04$ & $* * *$ \\
\hline \multirow[t]{7}{*}{ Dendritic cell } & CDIC & 0.36 & $1.3 \mathrm{E}-13$ & $* * *$ & -0.08 & $6.6 \mathrm{E}-0 \mathrm{I}$ & - \\
\hline & NRPI & 0.27 & $4.0 \mathrm{E}-08$ & $* * *$ & 0.01 & $9.5 \mathrm{E}-01$ & - \\
\hline & ITGAX & 0.10 & 4.4E-02 & $*$ & 0.31 & $6.9 \mathrm{E}-02$ & - \\
\hline & HLA-DPBI & 0.01 & 8.7E-0I & - & 0.47 & $4.0 \mathrm{E}-03$ & $* *$ \\
\hline & HLA-DQBI & -0.07 & I.4E-0I & - & 0.6 & I.7E-04 & $* * *$ \\
\hline & HLA-DRA & -0.08 & $9.9 \mathrm{E}-02$ & - & 0.59 & $2.2 \mathrm{E}-04$ & $* * *$ \\
\hline & HLA-DPAI & -0.02 & $6.8 \mathrm{E}-0 \mathrm{I}$ & - & 0.49 & $2.5 \mathrm{E}-03$ & $* *$ \\
\hline \multirow[t]{3}{*}{ Th2 cell } & STAT6 & 0.14 & 5. IE-03 & $* *$ & -0.35 & $3.6 \mathrm{E}-02$ & $*$ \\
\hline & STAT5A & 0.14 & $4.6 \mathrm{E}-03$ & $* *$ & -0.16 & $3.5 \mathrm{E}-0 \mathrm{I}$ & - \\
\hline & ILI3 & 0.06 & $2.0 \mathrm{E}-0 \mathrm{I}$ & - & -0.33 & $4.6 \mathrm{E}-02$ & $*$ \\
\hline \multirow[t]{4}{*}{ Treg } & FOXP3 & 0.14 & $4.8 \mathrm{E}-03$ & $* *$ & 0.46 & $5.0 \mathrm{E}-03$ & $* *$ \\
\hline & CCR8 & 0.16 & I.6E-03 & $* *$ & 0.28 & I.0E-0। & - \\
\hline & STAT5B & 0.32 & $5.5 \mathrm{E}-1 \mathrm{II}$ & $* * *$ & -0.31 & $6.3 \mathrm{E}-02$ & - \\
\hline & TGF $\beta($ TGFBI) & 0.20 & $3.3 \mathrm{E}-05$ & $* * *$ & -0.55 & $5.6 \mathrm{E}-04$ & $* * *$ \\
\hline \multirow[t]{3}{*}{ TAM } & CCL2 & 0.21 & 2.IE-05 & $* * *$ & -0.17 & $3.2 \mathrm{E}-0 \mathrm{I}$ & - \\
\hline & CD68 & 0.13 & $9.5 \mathrm{E}-03$ & $* *$ & 0.41 & I.4E-02 & $*$ \\
\hline & ILIO & 0.14 & 5.7E-03 & $* *$ & 0.38 & $2.2 \mathrm{E}-02$ & $*$ \\
\hline \multirow[t]{3}{*}{ M2 macrophage } & CDI63 & 0.01 & 9.IE-0I & - & -0.13 & $4.3 \mathrm{E}-0 \mathrm{I}$ & - \\
\hline & VSIG4 & 0.07 & $1.5 \mathrm{E}-0 \mathrm{I}$ & - & 0.15 & $3.8 \mathrm{E}-0 \mathrm{I}$ & - \\
\hline & MS4A4A & 0.12 & I.5E-02 & * & 0.07 & $6.9 \mathrm{E}-0 \mathrm{I}$ & - \\
\hline \multirow[t]{7}{*}{ NK cell } & KIR2DLI & -0.06 & 2.7E-05 & - & 0.25 & $1.5 \mathrm{E}-0 \mathrm{I}$ & - \\
\hline & KIR2DL3 & -0.08 & $\mathrm{I} .2 \mathrm{E}-0 \mathrm{I}$ & - & 0.30 & 7.3E-02 & - \\
\hline & KIR2DL4 & -0.21 & I.2E-05 & $* * *$ & 0.42 & I.0E-02 & $*$ \\
\hline & KIR3DLI & -0.08 & $9.1 \mathrm{E}-02$ & - & 0.21 & 2.IE-0I & - \\
\hline & KIR3DL2 & -0.06 & $2.0 \mathrm{E}-0 \mathrm{I}$ & - & 0.60 & I.2E-04 & $* * *$ \\
\hline & KIR3DL3 & -0.12 & I.3E-02 & $*$ & 0.14 & 4.IE-0I & - \\
\hline & KIR2DS4 & -0.11 & $2.6 \mathrm{E}-02$ & $*$ & 0.30 & $8.0 \mathrm{E}-02$ & - \\
\hline
\end{tabular}

Notes: P-value Significant Codes: $-P \geq 0.05, * P<0.05$, ** $P<0.01$, *** $P<0.001$.

EMT. ${ }^{41,42}$ Therefore, we presume that AGT may promote GC development by mediating EMT, while alterations in the immune microenvironment are also involved in this process, particularly in M2 macrophages. Extracellular matrix (ECM) is commonly composed of abundant type I fibrillar collagen, and ECM alterations in the tumor microenvironment are crosslinking for GC progression. ${ }^{43}$ Evidence confirms that ECM can enhance matrix stiffness, through enzyme- or non-enzymemediated pathways, and stiff ECM can lead to a negative effect on the malignancy of GC. ${ }^{44}$ Combined with the results of the GO analysis (collagen-containing extracellular matrix), we infer that AGT may promote GC progression by influencing ECM alterations.

This study does have some limitations. First, we obtained data from public databases, and our results may be influenced by the quality of data. For the purpose of decreasing the impact of data quality on the outcomes, multiple databases were used to evaluate the prognostic value of AGT expression in GC. Second, this study lacks in vivo and in vitro experiments, and the in-depth mechanism of AGT in GC needs to be further investigated. For instance, there is an effect of AGT overexpression on GC cell phenotypes, or regulatory mechanisms of aberrant AGT methylation in GC. 
A

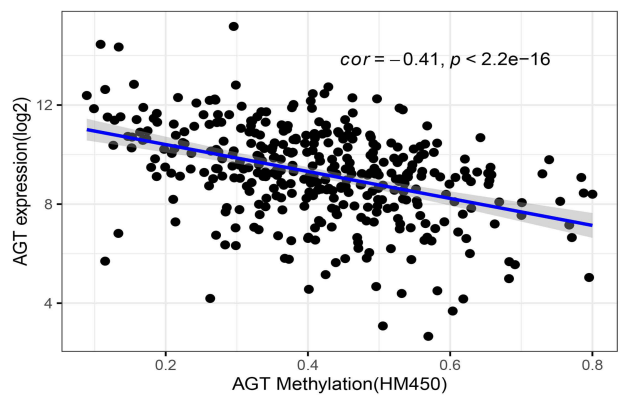

B

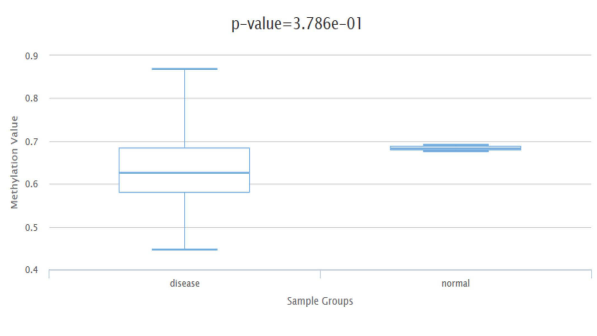

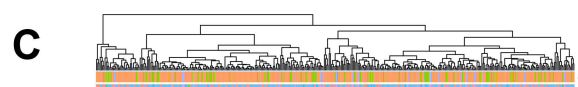
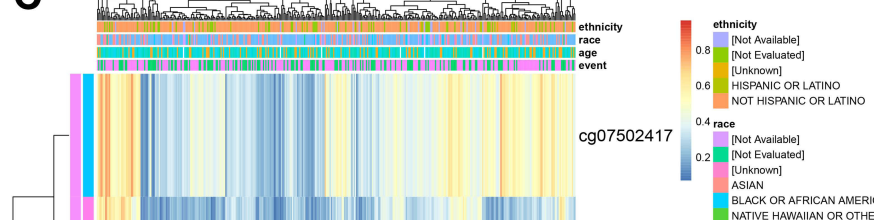

0.4 race
[Not Avalable]
[Not Evaluated]

[Not Evalual
[Unknown]
ASIAN

BLACK OR AFRICAN AMERICAN

$\operatorname{cg} 24474852$

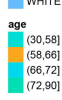

event
Alive
Dead

$\operatorname{cg} 22647018$

Relation_to_UCSC_CPG_Island
Open_Sea

UCSC_RefGene_Group

UcsC_RefGene_Grou
15tron:5UTR
SUTR
D
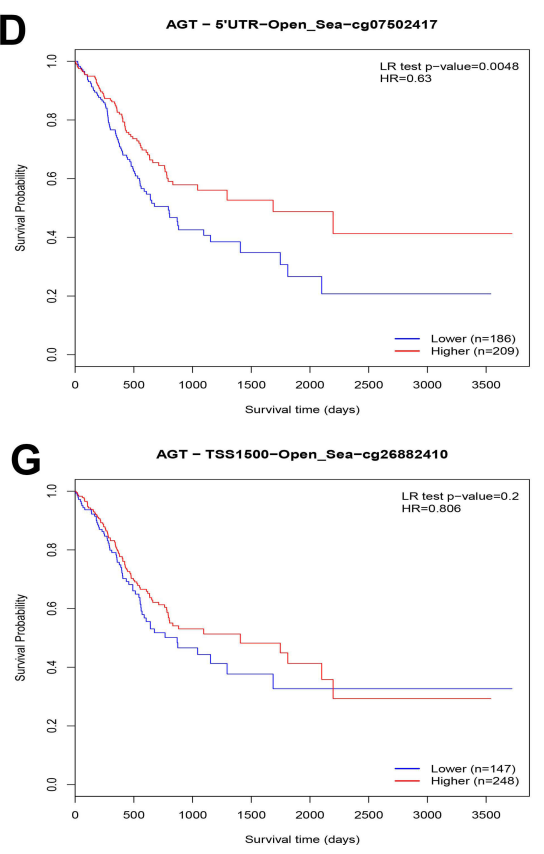

E

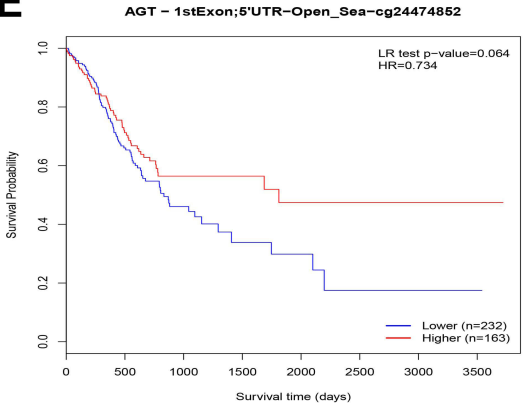

H

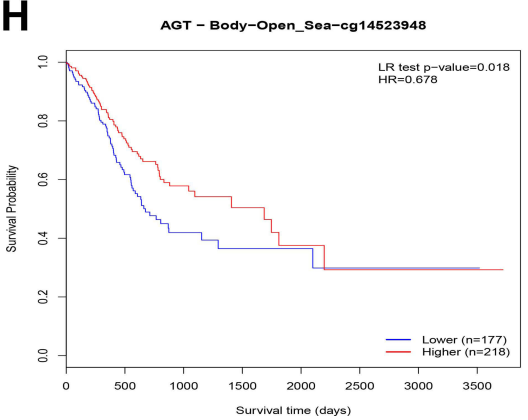

$\mathbf{F}$

F AGT-5'UtR-Open_Sea-cg22647018

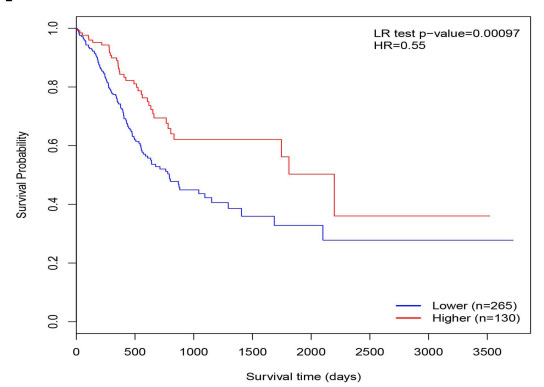

I

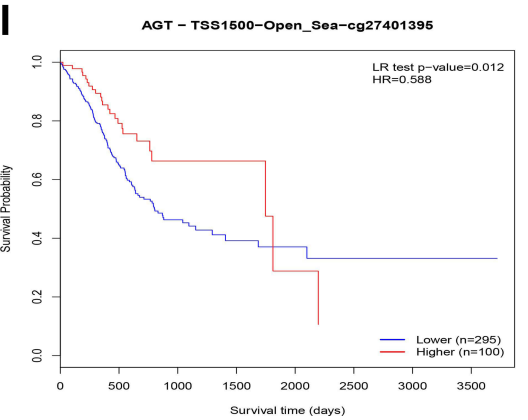

Figure 4 Methylation analysis of AGT. (A) Correlation between AGT mRNA expression and AGT DNA methylation. (B) AGT methylation between GC tissues and normal tissues. (C) Different methylated sites associated with AGT. The relationship between CpG sites and GC prognosis, including (D) cg075024I7; (E) cg24474852; (F) cg226470I8; (G) cg26882410; (H) cg14523948; (I) cg2740I395.

Abbreviation: HR, hazard ratio.

\section{Conclusion}

In conclusion, we found that abnormal upregulation of the AGT indicated poorer OS in GC especially in advanced GC. Increased AGT expression enhanced immune infiltration levels of macrophages, CD4+ T cells, and B cells in GC, and 
A

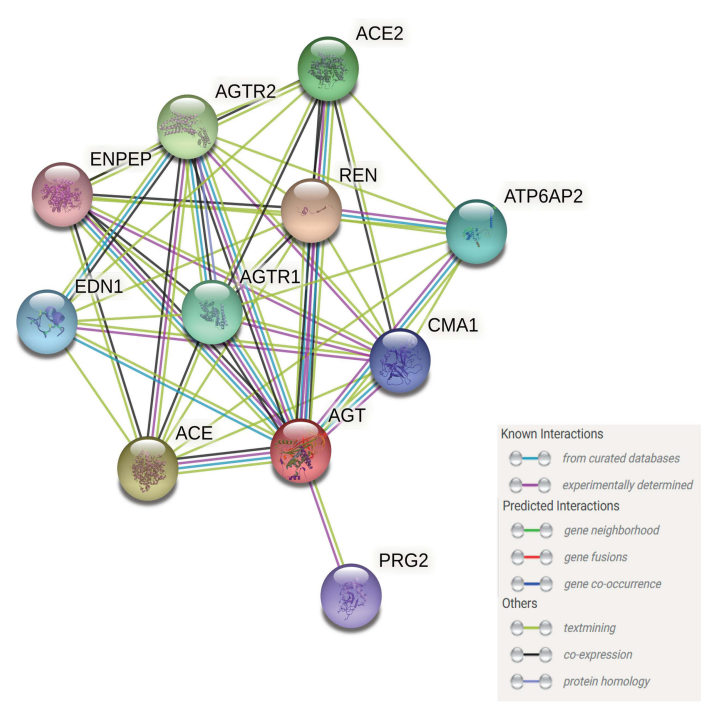

B

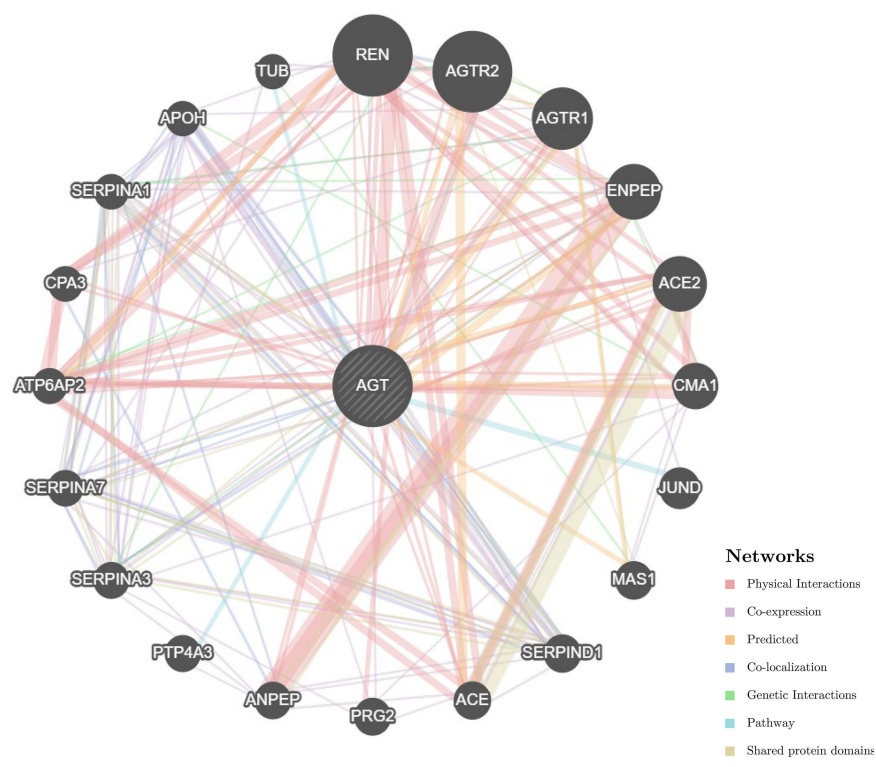

C

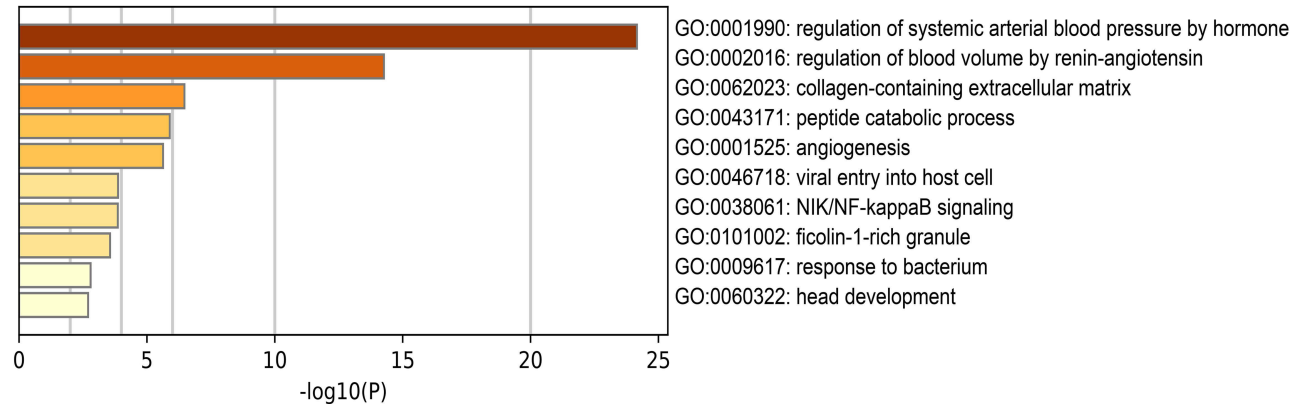

Figure 5 PPI network construction based on STRING database (A) and GeneMANIA database (B). GO analysis results (C).

AGT overexpression was associated with biomarkers of NK cells, TAMs, and Tregs, which could be a possible mechanism for AGT to influence GC patient outcomes.

\section{Abbreviations}

AGT, angiotensinogen; RAS, renin-angiotensin system; GC, gastric cancer; TCGA, The Cancer Genome Atlas; TIMER, Tumor Immune Estimation Resource; GEPIA, The Gene Expression Profiling Interactive Analysis; PPI, protein-protein interaction; GO, gene ontology; AFP, $\alpha$-fetoprotein; CEA, carcinoembryonic antigen; CA19-9, carbohydrate antigens 199; TME, tumor microenvironment; Ang I, angiotensin 1; Ang II, angiotensin 2; STAD, stomach adenocarcinoma; Th17, T-helper 17; Th1, T-helper 1; Th2, T-helper 2; Tfh, follicular helper T; NK, natural killer; OS, overall survival; RFS/DFS, recurrence-free survival/disease-free survival; STRING, The Search Tool for the Retrieval of Interacting Genes; AUC, the area under the receiver operating characteristic curve; ROC, receiver operating characteristic; DEGs, differentially expressed genes; HR, hazard ratio.

\section{Data Sharing Statement}

All data supporting the conclusion of this article are included within the article.

\section{Ethics Approval and Informed Consent}

This study was approved by Lanzhou University Second Hospital Medical Ethics Committee (PROJECT NO: 2021A596). All data in our study are from public databases. The patients involved in the databases have received ethical 
approval. Users can download relevant data for free to conduct research and publish relevant articles. Our study is based on open-source data, so Lanzhou University Second Hospital Medical Ethics Committee exempted us from the requirement of informed consent.

\section{Acknowledgments}

We are grateful to all researchers of enrolled studies.

\section{Funding}

This work was supported by the Industry support and guidance project of colleges and universities in Gansu Province (2019C-21), Cuiying Scientific and Technological Innovation Program of Lanzhou University Second Hospital - Key Cultivation Projects (CY2018-ZD01), and Cuiying Scientific Training Program for Undergraduates of Lanzhou University Second Hospital (CYXZ2021-54).

\section{Disclosure}

The authors report no conflicts of interest in this work.

\section{References}

1. Sung H, Ferlay J, Siegel RL, et al. Global Cancer Statistics 2020: GLOBOCAN Estimates of Incidence and Mortality Worldwide for 36 Cancers in 185 Countries. CA Cancer J Clin. 2021;71:209-249. doi:10.3322/caac.21660

2. Smyth EC, Nilsson M, Grabsch HI, van Grieken NC, Lordick F. Gastric cancer. Lancet. 2020;396:635-648. doi:10.1016/S0140-6736(20)31288-5

3. Chen C, Chen Q, Zhao Q, Liu M, Guo J. Value of Combined Detection of Serum CEA, CA72-4, CA19-9, CA15-3 and CA12-5 in the Diagnosis of Gastric Cancer. Ann Clin Lab Sci. 2017;47:260-263. doi:10.7314/apjcp.2015.16.9.3867

4. Xu Y, Zhang P, Zhang K, Huang C. The application of CA72-4 in the diagnosis, prognosis, and treatment of gastric cancer. Biochim Biophys Acta Rev Cancer. 2021;1876:188634. doi:10.1016/j.bbcan.2021.188634

5. Necula L, Matei L, Dragu D, et al. High plasma levels of COL10A1 are associated with advanced tumor stage in gastric cancer patients. World J Gastroenterol. 2020;26:3024-3033. doi:10.3748/wjg.v26.i22.3024

6. Yao Y, Ding Y, Bai Y, et al. Identification of Serum Circulating MicroRNAs as Novel Diagnostic Biomarkers of Gastric Cancer. Front Genet. 2020;11:591515. doi:10.3389/fgene.2020.591515

7. Guo X, Lv X, Ru Y, et al. Circulating Exosomal Gastric Cancer-Associated Long Noncoding RNA1 as a Biomarker for Early Detection and Monitoring Progression of Gastric Cancer: a Multiphase Study. JAMA Surg. 2020;155:572-579. doi:10.1001/jamasurg.2020.1133

8. Shan MJ, Meng LB, Guo P, et al. Screening and Identification of Key Biomarkers of Gastric Cancer: three Genes Jointly Predict Gastric Cancer. Front Oncol. 2021;11:591893. doi:10.3389/fonc.2021.591893

9. Hanahan D, Coussens LM. Accessories to the crime: functions of cells recruited to the tumor microenvironment. Cancer Cell. 2012;21:309-322. doi:10.1016/j.ccr.2012.02.022

10. Pei JP, Zhang CD, Yusupu M, Zhang C, Dai DQ. Screening and Validation of the Hypoxia-Related Signature of Evaluating Tumor Immune Microenvironment and Predicting Prognosis in Gastric Cancer. Front Immunol. 2021;12:705511. doi:10.3389/fimmu.2021.705511

11. Klein S, Duda DG. Machine Learning for Future Subtyping of the Tumor Microenvironment of Gastro-Esophageal Adenocarcinomas. Cancers. 2021;13(19):4919. doi:10.3390/cancers13194919

12. Akin Telli T, Bregni G, Camera S, et al. PD-1 and PD-L1 inhibitors in oesophago-gastric cancers. Cancer Lett. 2020;469:142-150. doi:10.1016/j. canlet.2019.10.036

13. Yang L, Shi P, Zhao G, et al. Targeting cancer stem cell pathways for cancer therapy.. Signal Transduct Target Ther. 2020;5(1):8. doi:10.1038/ s41392-020-0110-5

14. Matsusaka T, Niimura F, Shimizu A, et al. Liver angiotensinogen is the primary source of renal angiotensin II. J Am Soc Nephrol. 2012;23:1181-1189. doi:10.1681/ASN.2011121159

15. Lu H, Cassis LA, Kooi CW, Daugherty A. Structure and functions of angiotensinogen. Hypertens Res. 2016;39:492-500. doi:10.1038/hr.2016.17

16. Li S, Yu C, Cheng Y, Du F, Wen G. Bioinformatics analysis identifies biomarkers associated with poor prognosis in diffuse-type gastric cancer. Mol Med Rep. 2021;23:765. doi:10.3892/mmr.2021.11832

17. Wegman-Ostrosky T, Soto-Reyes E, Vidal-Millán S, Sánchez-Corona J. The renin-angiotensin system meets the hallmarks of cancer. $J$ Renin Angiotensin Aldosterone Syst. 2015;16:227-233. doi:10.1177/1470320313496858

18. Ames MK, Atkins CE, Pitt B. The renin-angiotensin-aldosterone system and its suppression. J Vet Intern Med. 2019;33:363-382. doi:10.1111/ jvim. 15454

19. Li XC, Zhang J, Zhuo JL. The vasoprotective axes of the renin-angiotensin system: physiological relevance and therapeutic implications in cardiovascular, hypertensive and kidney diseases. Pharmacol Res. 2017;125:21-38. doi:10.1016/j.phrs.2017.06.005

20. Catarata MJ, Ribeiro R, Oliveira MJ, Robalo Cordeiro C, Renin-Angiotensin MR. System in Lung Tumor and Microenvironment Interactions. Cancers. 2020;12:1457. doi:10.3390/cancers12061457

21. Shibata T, Tahara T, Arisawa T, Hirata I. Polymorphism of the salt sensitivity gene angiotensinogen and gastric cancer risk. Mol Med Rep. 2011;4:723-726. doi:10.3892/mmr.2011.495

22. Cui Y, Li Q, Li W, et al. NOTCH3 is a Prognostic Factor and Is Correlated With Immune Tolerance in Gastric Cancer. Front Oncol. 2020;10:574937. doi:10.3389/fonc.2020.574937 
23. Liu L, Pang H, He Q, et al. A novel strategy to identify candidate diagnostic and prognostic biomarkers for gastric cancer. Cancer Cell Int. 2021;21:335. doi:10.1186/s12935-021-02007-6

24. Zhang C, Liang Y, Ma MH, Wu KZ, Dai DQ. KRT15, INHBA, MATN3, and AGT are aberrantly methylated and differentially expressed in gastric cancer and associated with prognosis. Pathol Res Pract. 2019;215:893-899. doi:10.1016/j.prp.2019.01.034

25. Lu J, Huang XY, Wang YH, et al. POC1A acts as a promising prognostic biomarker associated with high tumor immune cell infiltration in gastric cancer. Aging. 2020;12:18982-19011. doi:10.18632/aging.103624

26. Wu T, Dai Y. Tumor microenvironment and therapeutic response. Cancer Lett. 2017;387:61-68. doi:10.1016/j.canlet.2016.01.043

27. Zhu H, Wang G, Zhu H, Xu A. ITGA5 is a prognostic biomarker and correlated with immune infiltration in gastrointestinal tumors. BMC Cancer. 2021;21:269. doi:10.1186/s12885-021-07996-1

28. Wu Y, Kuang DM, Pan WD, et al. Monocyte/macrophage-elicited natural killer cell dysfunction in hepatocellular carcinoma is mediated by CD48/ 2B4 interactions. Hepatology. 2013;57:1107-1116. doi:10.1002/hep.26192

29. Li T, Zhang Q, Jiang Y, et al. Gastric cancer cells inhibit natural killer cell proliferation and induce apoptosis via prostaglandin E2. Oncoimmunology. 2016;5:e1069936. doi:10.1080/2162402X.2015.1069936

30. Wynn TA, Chawla A, Pollard JW. Macrophage biology in development, homeostasis and disease. Nature. 2013;496:445-455. doi:10.1038/ nature 12034

31. Hu L, Zhang P, Sun W, et al. PDPN is a prognostic biomarker and correlated with immune infiltrating in gastric cancer. Medicine. $2020 ; 99:$ e19957. doi:10.1097/MD.0000000000019957

32. Liu X, Xu Q, Li Z, Xiong B. Integrated analysis identifies AQP9 correlates with immune infiltration and acts as a prognosticator in multiple cancers. Sci Rep. 2020;10:20795. doi:10.1038/s41598-020-77657-Z

33. Komohara Y, Fujiwara Y, Ohnishi K, Shiraishi D, Takeya M. Contribution of Macrophage Polarization to Metabolic Diseases. J Atheroscler Thromb. 2016;23:10-17. doi:10.5551/jat.32359

34. Liu Z, Kuang W, Zhou Q, Zhang Y. TGF- $\beta 1$ secreted by M2 phenotype macrophages enhances the stemness and migration of glioma cells via the SMAD2/3 signalling pathway. Int J Mol Med. 2018;42:3395-3403. doi:10.3892/ijmm.2018.3923

35. Li W, Zhang X, Wu F, et al. Gastric cancer-derived mesenchymal stromal cells trigger M2 macrophage polarization that promotes metastasis and EMT in gastric cancer. Cell Death Dis. 2019;10:918. doi:10.1038/s41419-019-2131-y

36. Whiteside TL. What are regulatory T cells (Treg) regulating in cancer and why? Semin Cancer Biol. 2012;22:327-334. doi:10.1016/j. semcancer.2012.03.004

37. Heyn H, Esteller M. DNA methylation profiling in the clinic: applications and challenges. Nat Rev Genet. 2012;13:679-692. doi:10.1038/nrg3270

38. Teng JJ, Zhao WJ, Zhang XL, et al. Downregulation of promoter methylation gene PRDM5 contributes to the development of tumor proliferation and predicts poor prognosis in gastric cancer. J Cancer. 2021;12:6921-6930. doi:10.7150/jca.59998

39. Corso G, Velho S, Paredes J, et al. Oncogenic mutations in gastric cancer with microsatellite instability. Eur J Cancer. 2011;47:443-451. doi:10.1016/j.ejca.2010.09.008

40. Fu ZH, Liu SQ, Qin MB, et al. NIK- and IKK $\beta$-binding protein contributes to gastric cancer chemoresistance by promoting epithelial-mesenchymal transition through the NF-אB signaling pathway. Oncol Rep. 2018;39:2721-2730. doi:10.3892/or.2018.6348

41. Yamaguchi T, Fushida S, Yamamoto Y, et al. Tumor-associated macrophages of the M2 phenotype contribute to progression in gastric cancer with peritoneal dissemination. Gastric Cancer. 2016;19:1052-1065. doi:10.1007/s10120-015-0579-8

42. Wang M, Li C, Liu Y, Wang Z. Effect of LAMA4 on Prognosis and Its Correlation with Immune Infiltration in Gastric Cancer. Biomed Res Int. 2021;2021:6428873. doi:10.1155/2021/6428873

43. Gilkes DM, Semenza GL, Wirtz D. Hypoxia and the extracellular matrix: drivers of tumour metastasis. Nat Rev Cancer. $2014 ; 14: 430-439$. doi:10.1038/nrc3726

44. Jang M, Koh I, Lee JE, et al. Increased extracellular matrix density disrupts E-cadherin/ $\beta$-catenin complex in gastric cancer cells. Biomater Sci. 2018;6:2704-2713. doi:10.1039/C8BM00843D

\section{Publish your work in this journal}

The International Journal of General Medicine is an international, peer-reviewed open-access journal that focuses on general and internal medicine, pathogenesis, epidemiology, diagnosis, monitoring and treatment protocols. The journal is characterized by the rapid reporting of reviews, original research and clinical studies across all disease areas. The manuscript management system is completely online and includes a very quick and fair peer-review system, which is all easy to use. Visit http://www.dovepress.com/testimonials.php to read real quotes from published authors.

Submit your manuscript here: https://www.dovepress.com/international-journal-of-general-medicine-journal 